\title{
DEFORMATION FINITENESS FOR REAL HYPERKÄHLER MANIFOLDS
}

\author{
Alex Degtyarev, Ilia Itenberg, Viatcheslav Kharlamov
}

\begin{abstract}
We show that the number of equivariant deformation classes of real structures in a given deformation class of compact hyperkähler manifolds is finite.
\end{abstract}

To Askold Khovansky with our admiration

\section{INTRODUCTION}

An irreducible holomorphic symplectic manifold is a Kählerian compact simply connected complex manifold $X$ such that the space $H^{0}\left(X, \Omega_{X}^{2}\right)$ is generated by a nowhere degenerate holomorphic 2 -form $\omega_{X}$.

In dimension two the only irreducible holomorphic symplectic manifolds are $K 3$ surfaces. Among examples in higher dimensions are the Hilbert schemes $\operatorname{Hilb}^{n}(X)$, where $X$ is a $K 3$-surface, and the generalized Kummer varieties $K^{n} T$, where $T$ is a complex two-dimensional torus (see $[\mathrm{Be}]$ ).

Sometimes, the irreducible holomorphic symplectic manifolds are also referred to as compact hyperkähler manifolds. More precisely, a compact hyperkähler manifold is an irreducible holomorphic symplectic manifold with a fixed Kähler class $\gamma_{X} \in H^{2}(X ; \mathbb{R})$. (Here and below, by a Kähler class we mean a cohomology class represented by the fundamental form of a Kähler metric.)

Recall that a real structure on a complex manifold $X$ is an anti-holomorphic involution conj: $X \rightarrow X$. An equivariant deformation is a Kodaira-Spencer family $p: \mathcal{X} \rightarrow B$ with real structures on both $\mathcal{X}$ and $B$ with respect to which the projection $p$ is equivariant. Sometimes we use the same term for the pull-back $p^{-1}\left(B_{\mathbb{R}}\right)$ of the real part of $B$. Clearly, locally the two notions coincide and, thus, give rise to the same equivalence relation.

A real structure on a compact hyperkähler manifold $\left(X, \gamma_{X}\right)$ is a real structure conj satisfying the additional property conj ${ }^{*} \gamma_{X}=-\gamma_{X}$. The usual averaging argument shows that each real irreducible holomorphic symplectic manifold admits a skew-invariant Kähler class, and two real compact hyperkähler manifolds are equivariantly deformation equivalent if and only if so are the underlying real irreducible holomorphic symplectic manifolds.

Our goal is to prove the following theorem.

1991 Mathematics Subject Classification. Primary: 53C26; Secondary: 14P25, 14J32, 32Q20.

The second and the third authors are supported by ANR-05-BLAN-0053-01 
1.1. Theorem. The number of equivariant deformation classes of real structures in a given deformation class of compact hyperkähler manifolds is finite.

This result exhibits what we call the deformation finiteness for real hyperkähler manifolds. As is known, a similar finiteness result holds for curves and surfaces. (Indeed, the only birational classes of surfaces for which the result is not found in the literature, either explicitly or implicitly, are elliptic surfaces and irrational ruled surfaces, but for the latter two classes the proof is more or less straightforward.) To our knowledge, almost nothing is known in higher dimensions.

Theorem 1.1 is inspired by the following finiteness result for complex hyperkähler manifolds, see Huybrechts [H2]: there exist at most finitely many deformation types of complex hyperkähler structures on a fixed underlying smooth manifold. Moreover, in many respects, out proof of Theorem 1.1 is similar to Huybrechts' proof of his complex statement. The crucial points remain the Kollár-Matsusaka finiteness theorem [KM], the Demailly-Paun characterization of the Kähler cone [DP], and the Calabi-Yau families.

Combining Theorem 1.1 with Huybrechts' statement cited above, one can replace the deformation class of complex manifolds in Theorem 1.1 with the diffeomorphism type of the underlying smooth manifold. Alternatively, one can consider the manifolds with a fixed Beauville-Bogomolov form $q$ and bounded constant $\lambda$, see Section 2 .

Note that a finiteness statement similar to Theorem 1.1 holds as well for equivariant deformation classes of holomorphic involutions (instead of real structures); the proof is literally the same as that of Theorem 1.1.

Acknowledgements. We are grateful to the Max-Planck-Institut für Mathematik and to the Mathematisches Forschungsinstitut Oberwolfach and its RiP program for their hospitality and excellent working conditions which helped us to accomplish an essential part of this work.

\section{Period SPACES}

For any irreducible holomorphic symplectic manifold $X$ there is a well defined primitive integral quadratic form $q_{X}: H^{2}(X ; \mathbb{Z}) \rightarrow \mathbb{Z}$ with the property that for some positive constant $\lambda \in \mathbb{R}$ (depending on the differential type of $X$ ) the identity $q_{X}(x)^{n}=\lambda\left(x^{2 n} \cap[X]\right)$ holds for any element $x \in H^{2}(X ; \mathbb{Z})$. (Here, the primitiveness means that $q_{X}$ is not a multiple of another integral form.) This quadratic form, called the Beauville-Bogomolov form, has inertia indexes $\left(3, b_{2}(X)-3\right)$ : it is positive definite on the subspace $\left(H^{2,0} \oplus H^{0,2}\right)_{\mathbb{R}} \oplus \gamma_{X} \mathbb{R}$ and negative definite on its orthogonal complement. Recall also that $H^{2,0} \oplus H^{0,2}$ and $H^{1,1}$ are orthogonal to each other with respect to the Beauville-Bogomolov form.

From now on, we fix a deformation class $\mathcal{D}$ of irreducible holomorphic symplectic manifolds. (Note that the property of being an irreducible holomorphic symplectic manifold is stable under Kähler deformations, see [B] , and that all sufficiently small deformations of a Kähler manifold are Kähler.) This defines the isomorphism type of the cohomology ring $H^{*}(X ; \mathbb{Z})$ and the Beauville-Bogomolov form $\left(H^{2}(X ; \mathbb{Z}), q_{X}\right)$. In particular, this fixes the constant $\lambda$ in the above description of the form.

In addition, we fix an abstract integral lattice $(L, q)$ isometric to $\left(H^{2}(X ; \mathbb{Z}), q_{X}\right)$. For a particular manifold $X \in \mathcal{D}$, a choice of an isometry $\varphi:\left(H^{2}(X ; \mathbb{Z}), q_{X}\right) \rightarrow$ $(L, q)$ is called a marking of $X$. 
Denote by Per the period domain

$$
\text { Per }=\{z \in L \otimes \mathbb{C} \mid q(z)=0, q(z+\bar{z})>0\} / \mathbb{C}^{*} \subset \mathbb{P}(L \otimes \mathbb{C}) .
$$

The period map, denoted by per, sends a marked manifold $(X, \varphi), X \in \mathcal{D}$, to the point $\operatorname{per}(X, \varphi)=\varphi\left(H^{2,0}(X)\right) \bmod \mathbb{C}^{*} \in$ Per. The period map is known to be surjective (see [H2]), and the following local Torelli theorem holds (see [B] ).

2.1. Theorem. Given a manifold $X \in \mathcal{D}$, there exists a universal (in the class of local deformations of $X$ ) local deformation

$$
p:\left(\mathcal{X}, \mathcal{X}_{0}=X\right) \rightarrow\left(\mathcal{B}, b_{0}\right) .
$$

Furthermore, for any marking $\varphi$ of $X$, the period map $B \rightarrow$ Per, $b \mapsto \operatorname{per} p^{-1}(b)$ (which is well defined due to the stability mentioned above), is a diffeomorphism of a neighborhood of the base point $b_{0}$ in $B$ to a neighborhood of its image $\operatorname{per}(X, \varphi)$ in Per.

Recall that a Kähler-Einstein metric on a manifold $X$ with complex structure $I$ is a Ricci flat $I$-invariant Riemannian metric $g$ on $X$ whose 'hermitization' $h(u, v)=$ $g(u, v)-i g(I u, v)$ is Kähler. The following fundamental statement is a well known corollary of the Calabi-Yau theorem.

2.2. Theorem. Let $\left(X, \gamma_{X}\right), X \in \mathcal{D}$, be a compact hyperkähler manifold. Then $\gamma_{X}$ is represented by a unique closed form $\rho_{I}$ such that the corresponding metric $g(u, v)=\rho_{I}(u, I v)$ is Kähler-Einstein with respect to the original complex structure $I$ and two additional complex structures $J$ and $K$ on $X$ satisfying the relation $I J=-J I=K$.

Consider a manifold $X \in \mathcal{D}$ and complex structures $I, J, K$ as in Theorem 2.2. For any triple $(a, b, c) \in S^{2}=\left\{\mathbf{x} \in \mathbb{R}^{3} \mid\|\mathbf{x}\|=1\right\}$, the operator $\lambda=a I+b J+c K$ is also a complex structure with respect to which $g$ is a Kähler-Einstein metric. Thus, a Kähler-Einstein metric on $X$ defines a whole 2-sphere of complex structures, each complex structure coming with a distinguished Kähler class. This sphere is naturally identified with the unit sphere in the (maximal) positive definite (with respect to $\left.q_{X}\right)$ subspace $V \subset H^{2}(X ; \mathbb{R})$ spanned by the corresponding Kähler classes $\gamma_{I}=\gamma_{X}, \gamma_{J}, \gamma_{K}$. Alternatively, $V$ is spanned by $\gamma_{X}, \operatorname{Re}\left[\omega_{X}\right]$, and $\operatorname{Im}\left[\omega_{X}\right]$ and, thus, depends on $X$ and $\gamma_{X}$ only. The corresponding sphere of complex structures is denoted by $\mathcal{S}\left(X, \gamma_{X}\right)$. Each element $\gamma \in \mathcal{S}\left(X, \gamma_{X}\right)$ is the Kähler class of a compact hyperkähler manifold (defined by $\gamma$ ) deformation equivalent to $\left(X, \gamma_{X}\right)$.

For any real compact hyperkähler manifold $\left(X, \gamma_{X}\right.$, conj), $X \in \mathcal{D}$, the involution conj $^{*}$ induced in the cohomology of $X$ is a $q_{X}$-isometry. Furthermore, conj ${ }^{*}$ interchanges $H^{2,0}$ and $H^{0,2}$, preserves $H^{1,1}$, and multiplies $\gamma_{X}$ by -1 . Therefore, with respect to $q_{X}$, the $(+1)$ - and $(-1)$-eigenlattices of conj $^{*}: H^{2}(X ; \mathbb{Z}) \rightarrow H^{2}(X ; \mathbb{Z})$ have positive inertia indexes 1 and 2, respectively.

The following statement is a straightforward consequence of Theorem 2.2 .

2.3. Corollary. Let $\left(X, \gamma_{X}\right.$, conj $), X \in \mathcal{D}$, be a real compact hyperkähler manifold, and let $\mathcal{S}_{\text {conj }}\left(X, \gamma_{X}\right) \subset \mathcal{S}\left(X, \gamma_{X}\right)$ be the circle fixed by conj*. Then each compact hyperkähler manifold defined by a class $\gamma \in \mathcal{S}_{\text {conj }}\left(X, \gamma_{X}\right)$ is real with respect to conj. 
Remark. Under the assumptions of Corollary 2.3, the holomorphic form $\omega_{X}$ on $X$ can be chosen so that conj ${ }^{*} \omega_{X}=\bar{\omega}_{X}$. With this choice, $\mathcal{S}_{\text {conj }}\left(X, \gamma_{X}\right)$ is the unit circle in the plane spanned by $\gamma_{X}$ and $\operatorname{Im}\left[\omega_{X}\right]$.

A real homological type is a $q$-isometry $c: L \rightarrow L$ whose $(+1)$-eigensublattice has positive inertia index 1 . Whenever $c$ is understood, we denote by $L_{ \pm}$its $( \pm 1)$ eigenlattices. The involution $c$ induces the map $\omega \mapsto \overline{c(\omega)}$ on the period space Per. Denote by $\operatorname{Per}(c)$ its fixed point set. Clearly, $\operatorname{Per}(c)$ consists of pairs $\left(\omega_{+} \mathbb{R}, \omega_{-} \mathbb{R}\right)$, where $\omega_{+} \in L_{+} \otimes \mathbb{R}, \omega_{-} \in L_{-} \otimes \mathbb{R}$, and $q\left(\omega_{+}\right)=q\left(\omega_{-}\right)>0$.

Fix a real homological type $c$. A $c$-marking of a real manifold $(X, \operatorname{conj}), X \in \mathcal{D}$, is a marking $\varphi:\left(H^{2}(X ; \mathbb{Z}), q_{X}\right) \rightarrow(L, q)$ commuting with $c$, i.e., such that $c \circ \varphi=$ $\varphi \circ$ conj $^{*}$. Clearly, the image of a $c$-marked manifold $(X, c o n j, \varphi)$ under the period map per belongs to $\operatorname{Per}(c)$.

2.4. Theorem. Any c-marked real manifold $(X, \operatorname{conj}, \varphi), X \in \mathcal{D}$, admits an equivariant local deformation over a base diffeomorphic to a neighborhood of the image $\operatorname{per}(X, \operatorname{conj}, \varphi)$ in $\operatorname{Per}(c)$.

Proof. Pick a universal local deformation $p:\left(\mathcal{X}, \mathcal{X}_{0}\right) \rightarrow\left(\mathcal{B}, b_{0}\right)$ of $\mathcal{X}_{0}=X$ given by Theorem 2.1. Due to the universality, the real structure conj: $X \rightarrow X$ extends to a unique fiber preserving anti-holomorphic map $\tilde{c}: \mathcal{X} \rightarrow \mathcal{X}$. On the other hand, one has $H^{0}\left(X ; \mathcal{T}_{X}\right)=H^{0}\left(X ; \Omega_{X}^{1}\right)=H^{1,0}(X)=0$, i.e., $X$ has no infinitesimal automorphisms. Hence, $\tilde{c}$ is an involution. The restriction of $p$ to the pull-back of $\operatorname{Per}(c)$ under the period map $b \mapsto \operatorname{per} p^{-1}(b)$ is the desired equivariant deformation.

Next statement is a corollary of the Demailly-Paun description $\sqrt{\mathrm{DP}}]$ of the Kähler cone; see [H1] for details.

2.5. Theorem. Let $\mathcal{K}_{X} \subset H^{2}(X ; \mathbb{R})$ be the Kähler cone of a manifold $X \in \mathcal{D}$, and let $\varphi$ be a marking of $X$. If the period $\omega=\operatorname{per}(X, \varphi)$ is generic, then the image $\varphi\left(\mathcal{K}_{X}\right)$ coincides with the positive cone in the hyperbolic space $\omega^{\perp} \cap(L \otimes \mathbb{R})$.

Remark. More precisely, 'generic' in Theorem 2.5 means the following (see [F]): no power of $\omega_{X}$ should annihilate an integral homology class of $X$ and, in addition, at most one point from each sphere of the form $\mathcal{S}(Y, \gamma), Y \in \mathcal{D}$, should be removed. This implies that the conclusion of Theorem 2.5 still holds for a generic real period, i.e., for any real homological type $c: L \rightarrow L$, the set of periods of real manifolds with maximal Kähler cone is dense in $\operatorname{Per}(c)$.

The last ingredient of the proof is the following statement due to J. Kollár and T. Matsusaka, see KM].

2.6. Theorem. For any integer $n>0$ and any pair of integers $a$ and $b$ there are universal constants $m, N$ such that for any compact complex $n$-manifold $X$ and any ample line bundle $\mathcal{L}$ on $X$ satisfying the relations $c_{1}(\mathcal{L})^{n}=a$ and $c_{1}(\mathcal{L})^{n-1} c_{1}(X)=$ $b$ the bundle $\mathcal{L}^{m}$ is very ample and $\operatorname{dim}\left|L^{m}\right| \leqslant N$.

2.7. Corollary. Fix a real homological type $c$ and a vector $l \in L_{-}$with $q(l)>0$. Then, the real compact hyperkähler manifolds $\left(X, \gamma_{X}\right.$, conj), $X \in \mathcal{D}$, admitting a c-marking $\varphi$ such that $\varphi\left(\gamma_{X}\right)=l$ constitute finitely many deformation families.

Proof. After a small perturbation, one can assume that the Picard group Pic $X=$ $\operatorname{per}(X, \varphi)^{\perp} \cap L$ is isomorphic to $\mathbb{Z}$. Then $\gamma_{X}=c_{1}(\mathcal{L})$ for some ample line bundle $\mathcal{L}$, and Theorem 2.7 applied to $a=l^{n}$ and $b=0$ gives constants $m, N$ such that the bundle $\mathcal{L}^{m}$ embeds $X$ to the projective space $\left|L^{m}\right|^{\vee}$ of dimension at most $N$, the 
degree of the image being $l^{n}$. Since $X$ is simply connected and the Chern class $c_{1}\left(\mathcal{L}^{m}\right)$ is conj ${ }^{*}$-skew-invariant, the real structure conj lifts to a real structure on $\mathcal{L}$, which descends to a real structure on $\left|\mathcal{L}^{m}\right|^{\vee}$ (possibly, nonstandard) with respect to which the above embedding is equivariant. Thus, the manifolds in question are realized as real submanifolds of bounded degree of real projective spaces of bounded dimensions; such manifolds form finitely many deformation families, each family consisting of compact hyperkähler manifolds due to the deformation stability.

\section{Proof of Theorem 1.1}

3.1. Lemma. Let $M$ be a lattice with at least two positive squares, and let $v_{1}, v_{2} \in$ $M \otimes \mathbb{R}$ be two vectors with $v_{1}^{2}>0$ and $v_{2}^{2}>0$. Then there exists a vector $v \in M \otimes \mathbb{R}$ such that the bilinear form is positive definite on both the plane generated by $v$ and $v_{1}$ and the plane generated by $v$ and $v_{2}$.

Proof. Consider the subspace $V$ generated by $v_{1}$ and $v_{2}$. If it has two positive squares, take $v \in V$; otherwise, $V^{\perp}$ has a positive square, and take $v \in V^{\perp}$.

3.2. Proof of Theorem 1.1. Up to isomorphism, $(L, q)$ admits at most finitely many involutive isometries $c: L \rightarrow L$. (Indeed, enumerating involutive isometries of a lattice reduces to enumerating isomorphism classes of lattices of bounded determinant, $c f . \sqrt[\mathbb{N}]{ }$, and their number is finite, see $\mathbb{C}$.) Thus, it suffices to show that each real homological type $c$ is realized by finitely many deformation families.

Fix $c$ and pick a class $l \in L_{-}$with $q(l)>0$. We will show that any $c$-marked real compact hyperkähler manifold $\left(X, \gamma_{X}, \operatorname{conj}, \varphi\right), X \in \mathcal{D}$, is equivariantly deformation equivalent to a $c$-marked real compact hyperkähler manifold $\left(Y, \gamma_{Y}\right.$, conj, $\left.\psi\right)$ with $\psi\left(\gamma_{Y}\right)=l$. Due to Corollary 2.7, manifolds $Y$ with this property constitute finitely many deformation families.

Consider a $c$-marked real compact hyperkähler manifold $\left(X, \gamma_{X}, \operatorname{conj}, \varphi\right), X \in \mathcal{D}$. Let $\gamma=\varphi\left(\gamma_{X}\right) \in L_{-} \otimes \mathbb{R}$, and denote by $\omega_{ \pm} \in L_{ \pm} \otimes \mathbb{R}$ the periods of $X$. To construct the desired deformation, we use the following three moves of the triple $\left(\omega_{+}, \omega_{-}, \gamma\right)$ in the period space:

(1) a rotation in the plane spanned by $\omega_{-}$and $\gamma$,

(2) a small perturbation of $\omega_{+}$in $L_{+} \otimes \mathbb{R}$ and of $\omega_{-}$and $\gamma$ in $L_{-} \otimes \mathbb{R}$, so that $\omega_{-}$and $\gamma$ remain orthogonal to each other,

(3) a move of $\gamma$ within the cone $\varphi\left(\mathcal{K}_{X}\right) \cap\left(L_{-} \otimes \mathbb{R}\right)$, where $\mathcal{K}_{X} \subset H^{2}(X ; \mathbb{R})$ is the Kähler cone of $X$.

Moves (11) and (2) are followed by equivariant deformations of $\left(X, \gamma_{X}\right.$, conj $)$ due to Corollary 2.3 and Corollary 2.4, respectively. For move (3), if the pair $\left(\omega_{+}, \omega_{-}\right)$is sufficiently generic, Theorem 2.5 implies that $\gamma$ can vary within the whole positive cone in the hyperbolic space $\omega_{-}^{\perp} \subset L_{-} \otimes \mathbb{R}$.

It remains to start with $\left(\omega_{ \pm}^{(0)}, \gamma^{(0)}\right)=\left(\omega_{ \pm}, \gamma\right)$ and construct a sequence of triples $\left(\omega_{ \pm}^{(i)}, \gamma^{(i)}\right), 0 \leqslant i \leqslant k$, each obtained from the previous one by one of the three moves, so that $\gamma^{(k)}=l$. As explained above, that would give rise to a sequence of $c$ marked compact hyperkähler manifolds $\left(X^{(i)}, \gamma_{X^{(i)}}, \operatorname{conj}, \varphi\right)$ deformation equivalent to each other, and one can take $\left(Y, \gamma_{Y}, \operatorname{conj}, \psi\right)=\left(X^{(k)}, \gamma_{X^{(k)}}, \operatorname{conj}, \varphi\right)$.

The desired sequence can be constructed as follows. First, perturb $\left(\omega_{ \pm}^{(0)}, \gamma^{(0)}\right)$ to a generic triple $\left(\omega_{ \pm}^{(1)}, \gamma^{(1)}\right)$ (move (2)). Apply Lemma 3.1 to get a vector $v \in L_{-} \otimes \mathbb{R}$ such that both the plane spanned by $v$ and $\omega_{-}^{(1)}$ and the plane spanned by $v$ and $l$ 
are positive definite. Since the periods $\omega_{ \pm}^{(1)}$ are generic, one can replace $\gamma^{(1)}$ with a vector $\gamma^{(2)}$ in the plane spanned by $v$ and $\omega_{-}^{(1)}$ (move (3)). Let $\omega_{ \pm}^{(2)}=\omega_{ \pm}^{(1)}$, use move (1) to produce a triple $\left(\omega_{ \pm}^{(3)}, \gamma^{(3)}\right)$ with $\omega_{-}$a positive multiple of $v$, and perturb it to a generic triple $\left(\omega_{ \pm}^{(4)}, \gamma^{(4)}\right)$ (move (2)) so that the plane spanned by $\omega_{-}^{(4)}$ and $l$ is still positive definite. Finally, replace $\gamma^{(4)}$ with a vector $\gamma^{(5)}$ in the plane spanned by $\omega_{-}^{(4)}$ and $l$ and such that $q\left(\gamma^{(5)}\right)=q(l)$ (move (3)), let $\omega_{ \pm}^{(5)}=\omega_{ \pm}^{(4)}$, and use move (1) to produce a triple $\left(\omega_{ \pm}^{(6)}, \gamma^{(6)}\right)$ with $\gamma^{(6)}=l$.

\section{REFERENCES}

[Be] A. Beauville, Variétés Kähleriennes dont la première classe de Chern est nulle, J. Diff. Geom. 18 (1983), 755-782.

[Bo] F. A. Bogomolov, Hamiltonian Kähler manifolds, Sov. Math. Dokl. 19 (1978), 1462-1465.

[DP] J.-P. Demailly, M. Paun, Numerical characterization of the Kähler cone of a compact Kähler manifold, Ann. of Math. 159 (2004), 1247-1274.

[C] J. W. S. Cassels, Rational Quadratic Forms, Academic Press, New York, London, 1978.

[F] A. Fujiki, On the de Rham Cohomology Group of a Compact Kähler Symplectic Manifold, Adv. Stud. Pure Math. 10 (1987), 105-165.

[H1] D. Huybrechts, Erratum: Compact hyperkähler manifolds: basic results, Preprint math. AG/0106014.

[H2] D. Huybrechts, Finiteness results for compact hyperkähler manifolds, J. reine angew. Math. 558 (2003), 15-22.

[KM] J. Kollár, T. Matsusaka, Riemann-Roch type inequalities, Amer. J. Math. 105 (1983), 229252.

[N] V. V. Nikulin, Integer quadratic forms and some of their geometrical applications, Izv. Akad. Nauk SSSR, Ser. Mat 43 (1979), 111-177 (Russian); English transl. in Math. USSRIzv. 43 (1979), 103-167.

[S] Y.-T. Siu, Every K3 surface is Kähler, Invent. Math. 73 (1983), 130-150.

BiLKENT UNIVERSity

06800 Ankara, Turkey

E-mail address: degt@fen.bilkent.edu.tr

Université Louis Pasteur et IRMA (CNRS)

7 rue René Descartes 67084 Strasbourg Cedex, France

E-mail address: itenberg@math.u-strasbg.fr

Université Louis Pasteur et IRMA (CNRS)

7 rue René Descartes 67084 Strasbourg Cedex, France

E-mail address: kharlam@math.u-strasbg.fr 